\title{
Simulation of the fluid structure interaction for an aerostatic bearing and a flexible substrate
}

\author{
R. Olieslagers ${ }^{1}$, M. de Wild ${ }^{2}$, S. van Melick ${ }^{1,3}$ \& R. Knaapen ${ }^{1}$ \\ ${ }^{I}$ TNO Science and Industry, The Netherlands \\ ${ }^{2}$ Department of Turbulence and Vortex Dynamics, \\ University of Eindhoven, The Netherlands \\ ${ }^{3}$ TMC Mechanical, The Netherlands
}

\begin{abstract}
The fluid structure interaction for an aerostatic bearing and a substrate is solved numerically by a semi-analytical model, programmed in the software package MATLAB. This semi-analytical model uses a fluidic network of resistances and capacities to solve the pressure field in the bearing channel. These pressures are sent to a mechanical module, which computes the substrate deformations by the direct stiffness method. This semi-analytical model is verified by a second model, built into the commercial software package ANSYS. The ANSYS model includes a two-way coupled FEM and FVM solver. Position and time-dependent bearing height variations are computed by means of a dynamic mesh. The implementation of the semi-analytical model is done and verified by three cases. The first case verifies the pressure profile inside a parallel plate configuration with a moving top wall. The last two cases verify the time-dependent position of a rigid and flexible substrate supported by an aerostatic bearing. The semianalytical model is proved to be an effective tool for aerostatic bearing design, since it is able to solve the FSI within a couple of minutes instead of days for a coupled FEM and FVM solver

Keywords: aerostatic bearings, air film squeeze flow, fluid structure interaction, web handling, Euler Bernoulli theory, discrete stiffness method.
\end{abstract}

\section{Introduction}

In high precision equipment for semiconductor manufacturing, aerostatic bearings are widely used for several functions in which frictionless or contactless 
motion of machine components is required. TNO is increasingly applying aerostatic bearing technology in equipment for Spatial Atomic Layer Deposition (Spatial-ALD). This Spatial ALD equipment is capable to produce ultrathin conformal coatings with an atomic level control over thickness in high throughput applications (e.g. Poodt et al. [1]). In Spatial ALD equipment two reactants are separated spatially instead of time through sequential purge steps. Aerostatic bearing technology is here used for gas separation and contactless motion of the substrate with respect to the deposition unit (e.g. Poodt et al. [2]). In recent applications the substrate flexibility has increased significantly, causing substrate deformations in the order of the bearing channel height. For the design of next generation spatial ALD equipment it is desired to compute the fluid structure interaction, between the substrate and bearing gas, by an efficient semianalytical model. The paper is divided in five sections. The most relevant physics like gas bearing stiffness, gas film squeeze are introduced in the second section. The semi-analytical model will be explained in the third section. The last two sections show the results of the applied test cases and a brief conclusion.

\section{Theory}

\subsection{Principle of an aerostatic bearing}

The principle of an aerostatic bearing consists of supplying external pressurised gas between the bearing surfaces, in our application the fixed wall and a flexible or rigid substrate (e.g. van Beek [3]). The gas in general passes subsequently an inlet, bearing and an outlet channel (as outlined in Figure 1). The pressure in the bearing channel prevents contact between the fixed wall and the substrate. The substrate is kept near the fixed wall by an external load applied on the top side of the substrate. In terms of dynamics, an aerostatic bearing can be seen as a coupled mass- spring- damper system. Here the springs represent the stiffness of the aerostatic bearing, which is equal to the pressure response in the bearing channel to a substrate displacement. The dampers represent the pressure response in the bearing channel as result of the substrate velocity, which squeezes the gas in the bearing channel.

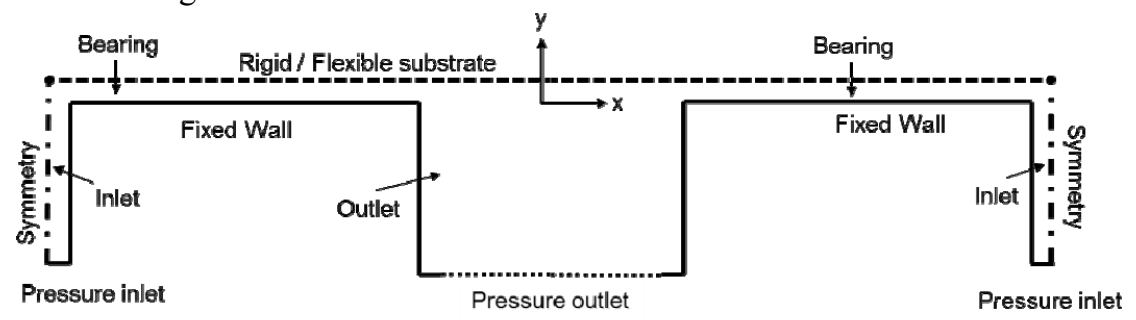

Figure 1: Model geometry of an aerostatic bearing.

\subsection{Bearing stiffness}

An aerostatic bearing exhibits stiffness by the gas flow through an inlet, bearing and outlet channel with a high, variable and low fluidic resistance respectively 
(Wesselingh [4]). If the substrate for example moves towards the fixed wall, the total resistance increases as result of the increasing bearing channel resistance. The fixed supply pressure and higher total resistance cause the mass flow to decrease. As result of the decreased mass flow, the pressure difference over the dominant inlet restriction drops, causing the bearing pressure to rise. The same reasoning can be made for a substrate moving away from the fixed wall. The stiffness of an aerostatic bearing gives the net force change in response of a height change.

\subsection{Gas film squeeze damping and gas compression}

The vertical (y) displacement of the substrate can be damped as result of gas film squeeze damping (e.g. Schrag and Wachutka [5]). The displacement of the substrate adds or extracts mass to the channel between the substrate and fixed wall as shown in Figure 2. For low substrate velocities the gas will have sufficient time to leak, resulting in a parabolic pressure profile in flow direction, which damps the substrate movement. However, for large substrate velocities, the gas will be compressed, resulting in a uniform pressure increase. This compression of gas behaves as an additional spring, since the uniform pressure change depends on the substrate displacement.

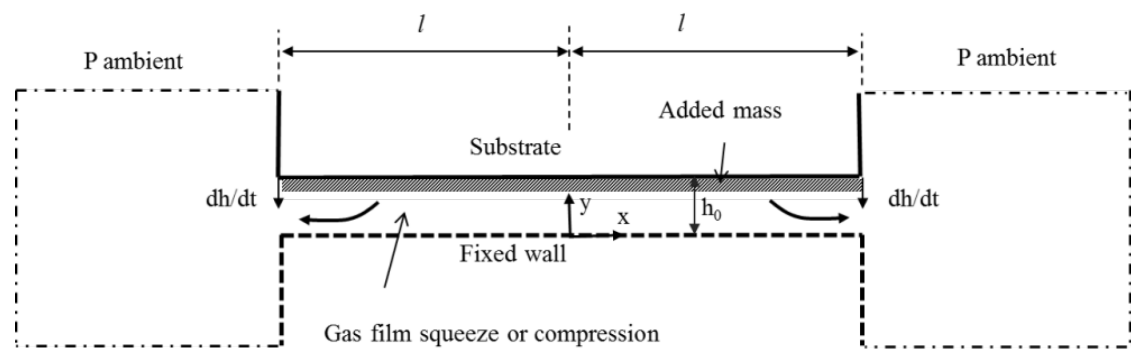

Figure 2: Model geometry used to study gas film squeeze in a channel with a moving top wall.

For small displacements $d h$ of the substrate around its equilibrium position $h_{o}$ the linearized and non-dimensional form of the Reynolds Eqn. (1) can be used to distinct a situation of gas compression from gas film squeeze (Bao and Yang [6]).

$$
\begin{gathered}
\frac{\partial^{2} \bar{p}}{\partial \bar{x}^{2}}-\sigma \frac{\partial \bar{p}}{\partial \bar{t}}=\sigma \frac{d \bar{h}}{d \bar{t}} \\
\sigma=\frac{12 \mu \omega l^{2}}{h_{0}^{2} p_{a}}
\end{gathered}
$$

in which $\mu$ is the dynamic viscosity, $\omega$ the oscillation frequency and $l$ the distance from the centre towards the outlet. The pressure $p$ is normalized by 
the ambient pressure $p_{a}, \bar{p}=p / p_{a}$, by using normalized variables, $\bar{x}=x / l$, $\bar{t}=\omega t, \bar{h}=h / h_{0}$. The degree of gas compression is related to the non dimensionless squeeze number $\sigma$, given by eqn. (2). For a very slow squeeze action or a squeeze number much smaller than unity, the gas will have sufficient time to leak. Under this condition eqn. (1) will reduce to eqn. (3)

$$
\frac{\partial^{2} \bar{p}}{\partial \bar{x}^{2}}=\sigma \frac{d \bar{h}}{d \bar{t}}
$$

In the case of a pure flow in x-direction, like in our application, eqn. (3) can be solved analytically as shown by eqn. (4). This equation shows a parabolic pressure profile, since increasingly more mass is added towards the outlets. The magnitude of the pressure profile depends on the velocity of the substrate, showing that the squeezed gas damps the displacement of the substrate.

$$
p(x)=\frac{6 \mu \frac{d h}{d t}}{h_{0}^{3}}\left(x^{2}-l^{2}\right)+p_{a}
$$

For very large squeeze numbers eqn. (1) reduces to eqn. (5)

$$
d \bar{p}=-d \bar{h} \Leftrightarrow p_{a} d h=-h_{0} d p
$$

This relation shows that the gas is fully compressed and is not able to leak. Here the pressure magnitude depends on the displacement of the substrate, showing that the aerostatic bearing will not damp the displacement of the substrate.

\section{Semi-analytical model}

The semi-analytical model, programmed in the software package MATLAB, consists of a fluidic and mechanical module. As result of multiple coupling iterations between the mechanical and fluidic module, the two-way coupled fluid structure interaction of the bearing channel and substrate is solved.

\subsection{Fluidic module}

The fluidic module computes the pressure $p$ and mass flow $\dot{m}$, in a network of coupled resistances $R$ and capacities $C a$ as shown in Figure 3. The configuration of this network corresponds to the aerostatic bearing geometry shown in Figure 1. One bearing channel is modelled by three in line positioned resistancecapacity-resistance blocks, depicted by the boxed area in Figure 3. The length $L$ of the boxed area corresponds to one element as shown in Figure 4. 


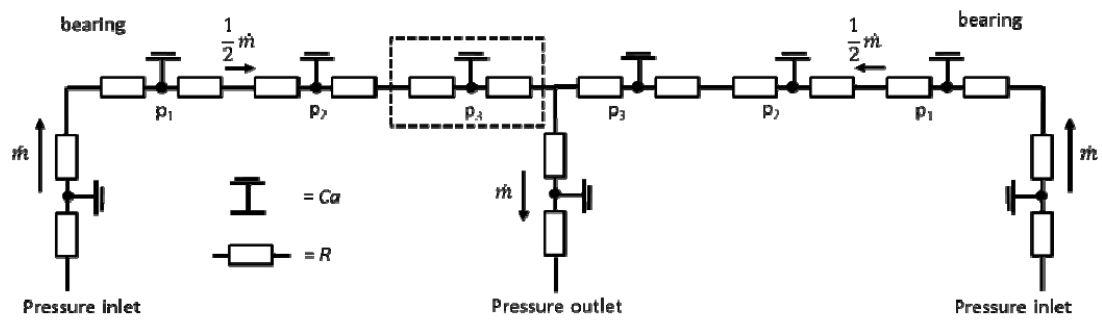

Figure 3: Fluidic network of an aerostatic bearing.

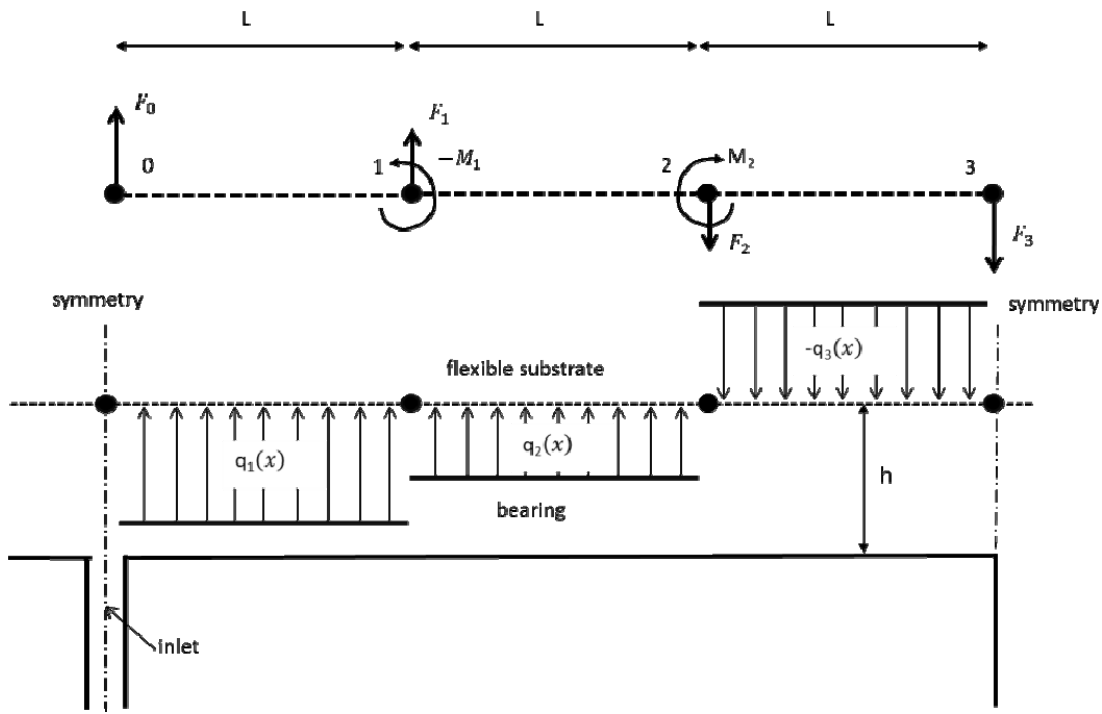

Figure 4: Discrete stiffness method applied on a flexible substrate.

The time dependent capacity pressure $d p / d t$ depends on the net mass flow and volume change of the capacity, as shown by eqn. (6) which is derived from the ideal gas law, assuming a constant temperature.

$$
\frac{d p}{d t}=\frac{R_{s} T}{V}\left(\dot{m}_{\text {in }}-\dot{m}_{\text {out }}\right)-\frac{m R_{s} T}{V^{2}} \frac{d V}{d t}
$$

where $R_{s}$ is the specific gas constant, $T$ the temperature of the gas, $V$ the volume of the capacity and $m$ the mass inside the capacity. The subscripts in and out correspond to an in or outflow of mass. The mass flows are calculated by eqn. (7) (van Beek [3]). 


$$
\dot{m}=\frac{p_{\text {left }}^{2}-p_{\text {right }}^{2}}{R}
$$

where $p_{\text {left }}$ and $p_{\text {right }}$ refer to the up and downstream pressure with respect to the resistance $R$. For a laminar flow in a channel in which the out of plane channel width is much larger than the height $w>>h$, the resistance $R$ is given by eqn. (8)

$$
R=\frac{12 \mu L R_{s} T}{w h^{3}}
$$

The capacity pressures $p_{1,} p_{2}$ and $p_{3}$ are transferred to the mechanical module. This mechanical module will convert the pressure $p$ to a load per unit length $q$ $\left(q_{i}=p_{i} * w\right)$ and uses the direct stiffness method to compute the substrate deformations.

\subsection{Mechanical module}

As the substrate flexibility increases in recent application, deformations need to be taken in account. The bending of the substrate is described by the EulerBernoulli beam theory, which is governed by eqn. (9).

$$
\frac{d^{2}}{d x^{2}}\left(E I \frac{d^{2} h}{d x^{2}}\right)=q
$$

where $E$ is the Young's modulus, $I$ is the second moment of area along the bending axis, $h$ the local height due deformations and $q$ the locally applied load per unit length. For simple distributions of $q$, a method known as the direct stiffness method can be used (Logan [7]). In this method the beam or in our application the substrate is divided in elements, with two nodes one on each end as shown in Figure 4. For a uniform load distribution on an element with a length $L$, the force $F_{i}$ and moment $M_{i}$ on a node are given by eqn. (10) and eqn. (11) respectively. The subscripts $i, j$ refer here to the node numbers. As result of the symmetry boundary conditions, no moment is applied on the nodes 0 and 3 .

$$
\begin{gathered}
F_{i}=\frac{\left(q_{i}+q_{i+1}\right) L}{2} \text { for } i=0,1,2,3 \\
M_{j}=-\frac{\left(q_{j}+q_{j+1}\right) L^{2}}{12} \text { for } j=1,2
\end{gathered}
$$

The force and moment on the nodes are put into one vector $\underline{F}$, so that $\underline{F}=\left(F_{0} ; F_{1} ; M_{1} ; F_{2} ; M_{2} ; F_{3}\right)$. The time dependent displacement and rotation of each element is computed by the force balance eqn. (12)

$$
\underline{F}=\underline{K} \cdot \underline{X}+\underline{M} \cdot \underline{\ddot{X}}
$$

where $\underline{X}=\left(d h_{0} ; d h_{1} ; \theta_{1} ; d h_{2} ; \theta_{2} ; d h_{3}\right)$ is the displacement vector consisting of the displacement $d h$ at the nodes and the angles of the elements with the 
horizontal axis $\theta$. The force balance eqn. (12) is solved for $\underline{X}$ by rearranging eqn. (12) into eqn. (13)

$$
\underline{X}=\underline{M}^{-1} \cdot(\underline{F}-\underline{K} \cdot \underline{X})
$$

The stiffness and mass matrix for one element is shown by eqn. (14) and eqn. (15) respectively.

$$
\begin{gathered}
\underline{K}=\frac{E I}{L^{3}}\left(\begin{array}{cccc}
12 & 6 L & -12 & 6 L \\
6 L & 4 L^{2} & -6 L & 2 L^{2} \\
-12 & -6 L & 12 & -6 L \\
6 L & 2 L^{2} & -6 L & 4 L^{2}
\end{array}\right)=\left(\begin{array}{ll}
{\left[k_{1}\right]} & {\left[k_{2}\right]} \\
{\left[k_{3}\right]} & {\left[k_{4}\right]}
\end{array}\right) \\
\underline{M}=\frac{\rho L w d}{420}\left(\begin{array}{cccc}
156 & 22 L & 54 & -13 L \\
22 L & 4 L^{2} & 13 L & -3 L^{2} \\
54 & 13 L & 156 & -22 L \\
-13 L & -3 L^{2} & -22 L & 4 L^{2}
\end{array}\right)=\left(\begin{array}{lll}
{\left[m_{1}\right]} & {\left[m_{2}\right]} \\
{\left[m_{3}\right]} & {\left[m_{4}\right]}
\end{array}\right)
\end{gathered}
$$

The previously not mentioned $\rho$ and $d$ are the density and thickness of the substrate elements. The global stiffness matrix for a substrate with three elements is found by applying superposition as shown by eqn. (16). The same method is applied to obtain the global mass matrix.

$$
\underline{K}_{\text {tot }}=\left(\begin{array}{cccc}
{\left[k_{1}\right]} & {\left[k_{2}\right]} & 0 & 0 \\
{\left[k_{3}\right]} & {\left[k_{4}\right]+\left[k_{1}\right]} & {\left[k_{2}\right]} & 0 \\
0 & {\left[k_{3}\right]} & {\left[k_{4}\right]+\left[k_{1}\right]} & {\left[k_{2}\right]} \\
0 & 0 & {\left[k_{3}\right]} & {\left[k_{4}\right]}
\end{array}\right)
$$

\section{Results}

The previously explained semi-analytical is verified by a model programmed in the software package ANSYS. The ANSYS model uses a FEM solver "ANSYS Mechanical" and a FVM solver "ANSYS CFX" to compute respectively the substrate deformation and flow field. The bearing channel is modelled by a dynamic mesh, with 15 and 350 quadrilateral cells in vertical (x) and horizontal direction (y). An upwind advection scheme and a first order Euler backward scheme is used to solve the time dependent flow field. The ANSYS Mechanical solver includes stiffness changes, since we expect deformations more than 10 percent of the substrate thickness (ANSYS [8]). The verification of the semianalytical model is done by three cases, shown in section 4.1 to 4.3 .

\subsection{Gas film squeeze}

The first case verifies the semi-analytical model for a gas film squeeze flow, in a channel with a prescribed substrate velocity as shown in Figure 2. The velocity of the substrate in $-\mathrm{y}$ direction is prescribed by an user defined function. The semi-analytical module uses nine in line positioned resistance-capacityresistance blocks and an ambient pressure on both ends of the channel. 


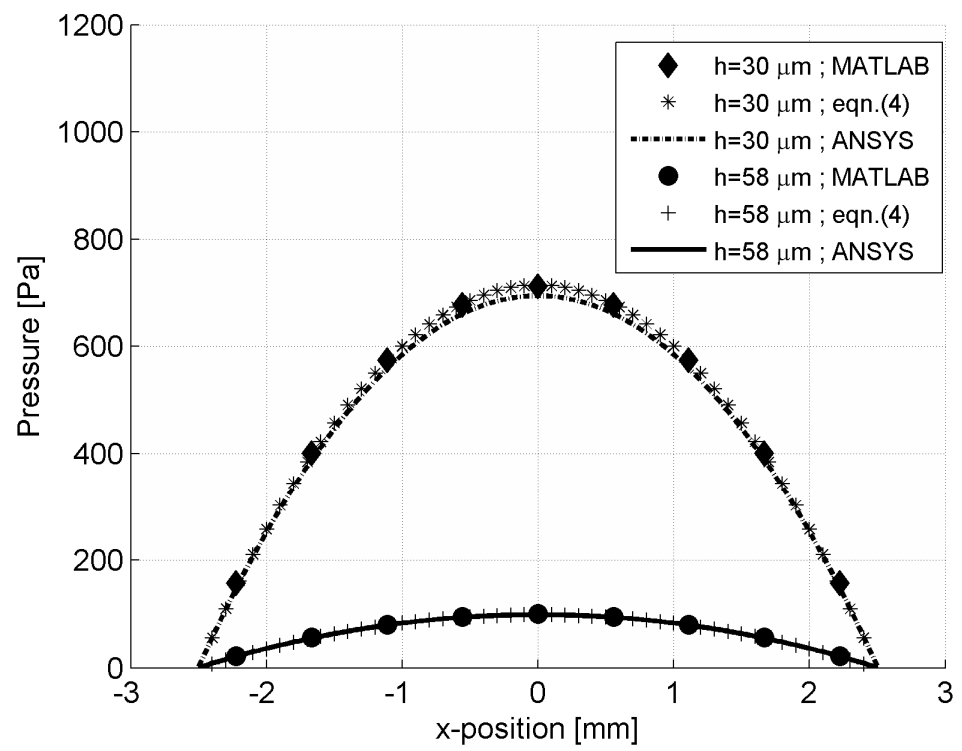

Figure 5: Pressure profile as result of gas film squeeze in a channel with a moving top wall.

The semi-analytical as well as the ANSYS model do not use a mechanical solver or module, since the velocity of the substrate is prescribed. Figure 5 shows the pressure profile as result of the downwards moving rigid substrate, with a velocity of $30 \mathrm{e}-3 \mathrm{~m} / \mathrm{s}$ for a channel height, $h$ of 30 and $58 \mu \mathrm{m}$. For this relative low substrate velocity, the semi-analytical and ANSYS model show a parabolic pressure distribution equal to eqn. (4). This shows that the gas has sufficient time to leak. In case of increased substrate velocities or high squeeze number lows, the semi-analytical model deviates from the ANSYS model, since inertia effects and wave equations are not implemented in the semi-analytical model.

\subsection{Aerostatic bearing and a rigid substrate}

The second case verifies the coupling of the bearing channel pressure field and a rigid substrate. Figure 1 shows the domain and boundary conditions of the ANSYS model. On both ends of the substrate, only a displacement in y direction is allowed. Rotations along $\mathrm{x}, \mathrm{y}$ and $\mathrm{z}$ axis are fixed at both ends. The semianalytical model uses the network configuration and discrete stiffness method as shown in Figures 3 and 4. In both models, substrate deformations are excluded, by setting the Young's modulus to an infinite high value. On the pressure inlet and outlet a fixed gauge pressure of $4 \mathrm{e} 3 \mathrm{~Pa}$ and $0 \mathrm{~Pa}$ was prescribed respectively. The simulation was initialized with a pressure of $0 \mathrm{~Pa}$ over the total domain and a bearing height of $200 \mu \mathrm{m}$. Figure 6 shows the bearing height or substrate position for a load pressure of 222 and $444 \mathrm{~Pa}$ respectively. The semi-analytical model as well as the ANSYS model show a decreasing substrate position to an 
asymptotic equilibrium height. The substrate settling velocity is approximately 25 to $50 \mathrm{~mm} / \mathrm{s}$, which indicates that the gas has sufficient time to leak.

Figure 7 shows the net pressure, which is the difference of the bearing and load pressure. The sum of the net pressure along $\mathrm{x}$ has decreased to zero, which

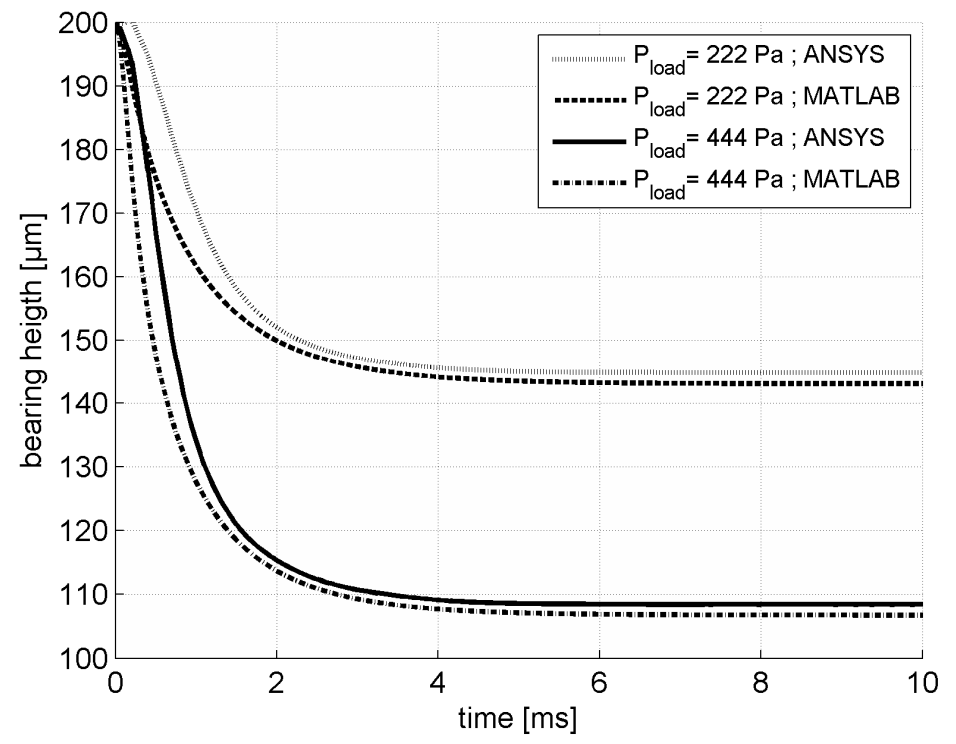

Figure 6: Bearing height as function of time.

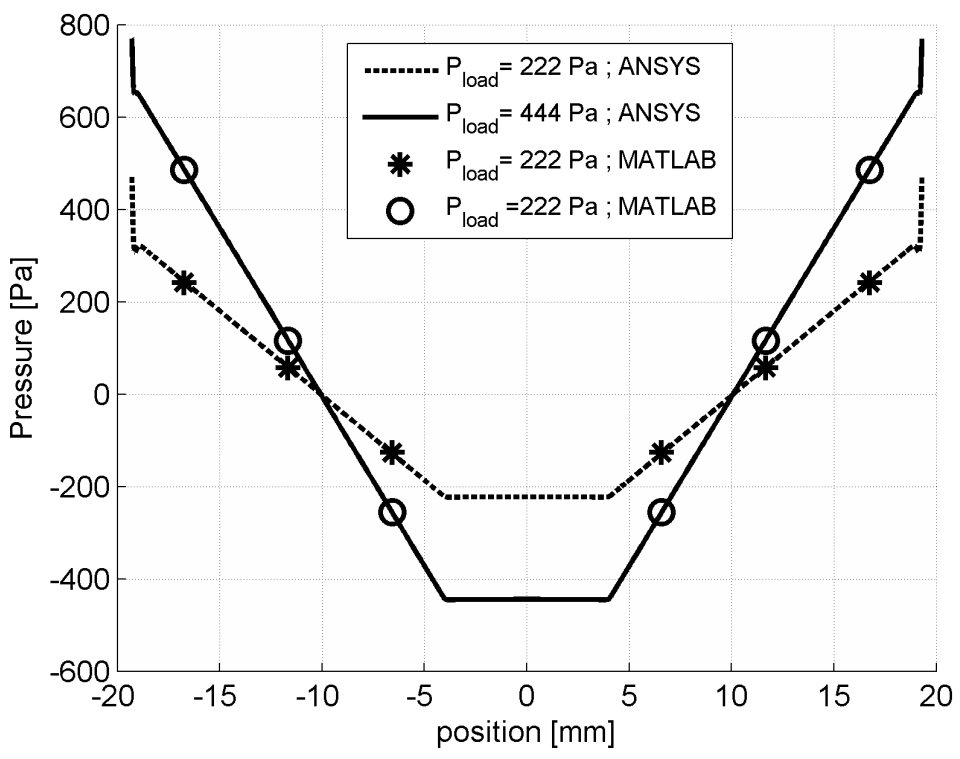

Figure 7: Bearing pressures at $\mathrm{t}=10 \mathrm{~ms}$. 
confirms that the substrate has settled to a steady state y position. The semianalytical model predicts an equilibrium substrate position within one percent of the ANSYS result, which is in general sufficiently accurate in aerostatic bearing design.

\subsection{Aerostatic bearing and a flexible substrate}

The last case verifies the coupling of the bearing channel pressure field and a flexible substrate. Both models described in section 4.2 use a Young's modulus of $3 \mathrm{e} 9 \mathrm{~Pa}$ for the substrate, which is equal to the modulus of PET, a commonly used substrate material in high tech industry. Figures 8 and 9 show the steady state substrate deformation and bearing pressure computed by the semi-analytical and ANSYS model. From qualitative point of view, both models show a similar substrate deformation. The bearing height decreases from a maximum height above the inlet towards a smaller height towards the outlet, which corresponds to the pressure profile shown in Figure 9. From quantitative perspective, the semianalytical model shows a larger substrate deformation. Most likely the semianalytical model over predicts the deformation, since stiffness effects as result of large deformations are not included. Future work will focus on the necessity to implement stiffness changes as result of large deformations in the semi-analytical model.

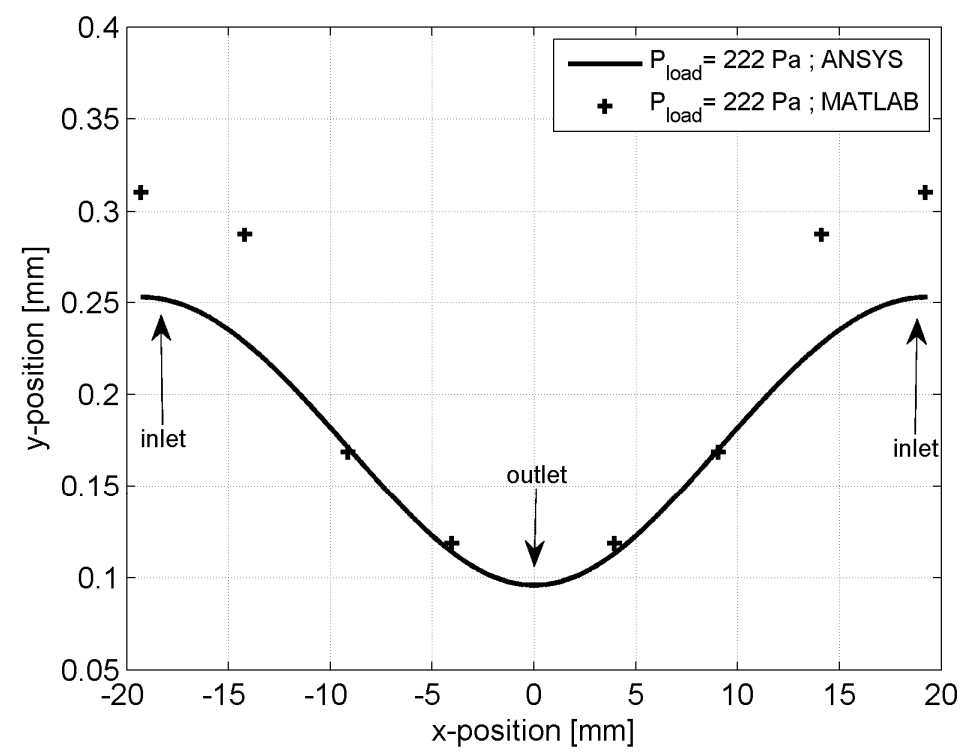

Figure 8: Steady state substrate deformation. 


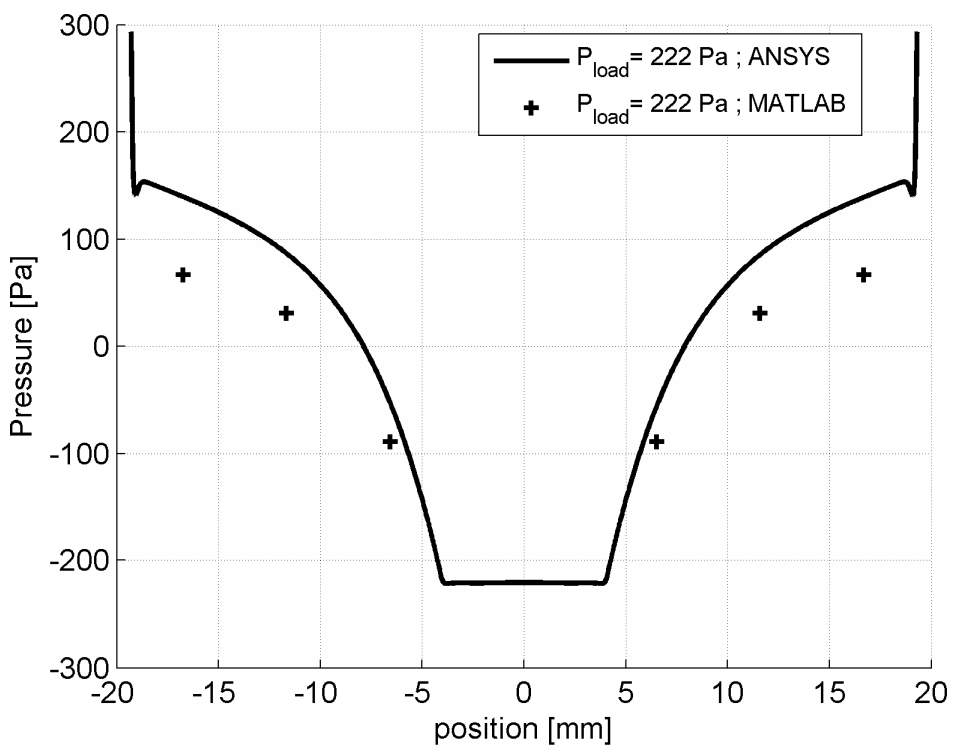

Figure 9: Steady state bearing pressure.

\section{Conclusion}

The two way fluid structure interaction for an aerostatic bearing and a substrate is solved numerically by a semi-analytic model and verified by an ANSYS model. For a rigid substrate, the semi-analytical and ANSYS model show a similar substrate position and pressure profile. The equilibrium substrate position computed by the ANSYS and semi-analytical model differ approximately 1 percent, which is in general sufficiently accurate for aerostatic bearing design. When the substrate is made flexible, both models show a qualitative similar substrate deformation. The over prediction of the substrate deformation by the semi-analytical model is most likely caused by stiffness effects as result of large substrate deformations. Future work will focus on the necessity to extend the semi-analytical model with stiffness effects as result of large substrate deformations. The semi-analytical model has become an effective tool in the design of aerostatic bearings, since it is able to solve the FSI within minutes instead of days for the coupled FEM and FVM solver.

\section{Acknowledgements}

The support given by A. Twerda, C. Rops and A. Ulucan is greatly appreciated. 


\section{References}

[1] Poodt P., Cameron D.C., Dickey E., George M.S., Kuznetsov V., Parsons G.N., Roozeboom F., Sundaram G., Vermeer, Spatial atomic layer deposition: A route towards further industrialization of atomic layer deposition, Journal of Vacuum Science and Technology, 30, 010802, 2012

[2] Poodt P., Lankhorst A, Roozeboom F., Spee K., Maas D., Vermeer A., High-Speed Spatial Atomic-Layer Deposition of Aluminum Oxide Layers for Solar Cell Passivation, Advanced Materials, 22, pp. 3564-3567, 2010

[3] van Beek A., Machine lifetime performance and reliability, Tribology-ABC, pp. 390-408, 2004

[4] Wesselingh J., Contactless positioning using an active film, IOP precisie technologie, pp 46-51, 2011

[5] Schrag G., Wachutka G., Physically based modelling of squeeze film damping by mixed-level system simulation, Sensors and Actuators, 97-98, pp. 193-200, 2002

[6] Bao M., Yang H., Squeeze film damping in MEMS, Sensors and Actuators, 136, pp. 3-27, 2007

[7] Logan, D.L., A first course in finite element method, Cengage Learning, pp. 31-66, 1992

[8] ANSYS Mechanical User Guide, ANSYS, Inc. 